\title{
Clinical implications of systemic lupus erythematosus without and with antiphospholipid syndrome in peri- and postmenopausal age
}

\author{
Bogna Grygiel-Górniak, Nattakarn Limphaibool, Mariusz Puszczewicz \\ Department of Rheumatology and Internal Medicine, Poznan University of Medical Sciences, Poznan, Poland
}

\begin{abstract}
Antiphospholipid syndrome (APS) in patients with systemic lupus erythematosus (SLE) displays a severe disease sub-phenotype with vascular manifestations ranging from peripheral thrombosis to neurologic and ophthalmic symptoms. The prevalence of morbidities including thrombosis, renal lesions, and cognitive impairment contributes to a higher risk of organ damage and a reduced quality of life in patients. In addition to the clinical heterogeneity, the diagnostic challenge is heightened in elderly patients as APS-related SLE is primarily diagnosed in young females. Many patients reach menopause due to the clinical association of premature menopause and improvements in diagnostic and therapeutic strategies in recent years. Although obstetric morbidity is not a concerning feature of the disease within this age group, a number of manifestations which may contribute to a decreased quality of life are present and must therefore not be disregarded. An improved prognosis derives from successful therapeutic regimens with minimal adverse effects in individual patients. The multifaceted management involves patient evaluation and risk stratification, followed by thromboprophylaxis efforts through the correction of modifiable risk factors, lifestyle recommendations, and pharmacological therapy. This review highlights the role of estradiol in the disease pathogenesis as well as the clinical complications and management of APS-related SLE in perimenopausal and postmenopausal patients.
\end{abstract}

Key words: antiphospholipid syndrome, estrogen, systemic lupus erythematosus, menopause.

\section{Introduction}

Antiphospholipid syndrome (APS) is defined by the occurrence of vascular thrombosis or pregnancy morbidity in the presence of positive laboratory findings of anti-cardiolipin antibody $(\mathrm{aCL})$, lupus anticoagulant, or anti-B2 glycoprotein-I antibody [1]. It is prevalent in up to $25 \%$ of patients with systemic lupus erythematosus (SLE), an autoimmune disease that remains a diagnostic challenge due to its diverse clinical manifestations [2]. The association of APS with SLE displays a severe disease sub-phenotype, with symptoms ranging from mild mucocutaneous involvement to severe pulmonary and cardiovascular disease [2, 3]. An increased risk of neuropsychiatric involvement and chronic renal insufficiency progression is also observed [1]. The increased mortality risk and reduced survival rate of patients are mainly attributable to the occurrence of thrombotic events [4].

In addition to the clinical heterogeneity, the diagnostic challenge is heightened in patients within an elderly age group who are not the typical demographic of the disease. Although obstetric morbidity is a concerning hallmark of the disease in women of reproductive age, it may involve many other manifestations that con- tribute to a decrease in quality of life. This review highlights the role of estradiol in the disease pathogenesis as well as the clinical complications and management of APS-related SLE in perimenopausal and postmenopausal patients.

\section{Epidemiology and prevalence}

Antiphospholipid syndrome-associated SLE primarily affects young females between the ages of 21 and 30 years. Many patients reach menopause due to recent advances in diagnostic and therapeutic strategies in addition to the increased frequency of premature menopause seen in the disease. The reported prevalence in the age groups $41-50$ and over 50 is $10.20 \%$ and $12.24 \%$ respectively $[3,5,6]$.

Differences exists across different geographic regions and ethnic groups in terms of both prevalence and presentation. The prevalence of APS in Caucasian patients with SLE is higher than that in the Chinese population. Amongst Chinese patients, arterial thrombosis was more frequently manifested than venous thrombosis and associated increased mortality risk was observed [4]. A number of clinical presentations were 
frequently found in Latin American patients including neurologic and dermatologic symptoms as well as hemolytic anemia [7].

Cervera et al., in a study of 1000 European APS patients, found that $36 \%$ of patients had APS-related SLE compared to $53 \%$ of patients with primary APS. Factors associated with aging could be responsible for the difference in the disease presentation. Early childhood onset is associated with increased episodes of jugular vein thrombosis and chorea in contrast to higher incidence of angina pectoris and stroke found in patients with a later disease onset [8].

The 5-year mortality rate observed in a cohort of European patients was $5.3 \%$. Thrombotic events including myocardial infarction and stroke along with infections and hemorrhagic complications contribute to one-third of the mortality rate. This is followed by malignancy, catastrophic APS, and pulmonary embolism, among others [8]. Antiphospholipid syndrome is associated with a high rate of morbidity and mortality in SLE patients. Therefore, prompt diagnosis and prophylactic treatment are critical to prevent fatal complications [3].

\section{Role of estrogen in disease pathogenesis}

The heterogeneity of APS-related SLE suggests the involvement of multiple pathogenic processes including environmental contributions which can affect the disease expression in genetically predisposed individuals $[7,9]$.

Similar to many other autoimmune diseases, a predominant preference of female patients and a primary disease onset during the reproductive years indicate a hormonal contribution to the disease pathogenesis [8]. The immunoregulatory properties of sex hormones have suggested that an interrelation exists between SLE disease activity and estradiol, though the mechanisms involved have not been fully explained [10, 11].

The role of estrogen in the promotion of autoimmunity is indicated as SLE disease susceptibility is associated with estrogen-related exposures (early menarche, pregnancy, exogenous estrogen therapy) [12]. Although this may indicate a possible association, it is not the sole factor, as SLE has also been reported in children, postmenopausal women, and in male patients. Theoretical links between estrogen and SLE have been built on the findings of increased disease activity with pregnancy or the menstrual cycle, increased estradiol levels in affected women, SLE association with estrogen receptor gene polymorphism (estrogen receptor alpha polymorphism), hormone replacement therapy (HRT)-associated mild-to-moderate flares, estrogen's effect on B-cell maturation, selection, and activity, and increased autoantibody production by estrogen [13, 14].

Results have shown that hyperestrogenic levels in SLE women are linked to an increased risk of APS and cardiovascular manifestation. Higher levels of estrogen were reported in SLE patients with APS compared to patients without APS. Estrogen was shown to contribute to the clinical manifestations as higher frequencies of thrombocytopenia were observed in patients with higher estradiol levels. A significant increase in episodes of transient ischemic attack and stroke was also reported compared to patients with normal estradiol levels. In addition to the thrombotic enhancement properties of estrogens in SLE patients, estrogens have also been implicated in stimulation of the pathologic type of $\mathrm{aCL}$. This demonstrates a potential important correlation between estrogens and autoimmunity, with an impact on the risk of thrombosis and cardiovascular disease [11]. Other factors that may affect the thrombotic-hemostatic balance in favor of thrombosis may include the presence of infection, endothelial injury, and factors such as a history of prior surgery and immobility [9] (Figure 1).

\section{Clinical manifestations}

A similar clinical profile between patients with primary APS and SLE-related APS was observed [8]. A variety of vascular manifestations across different organ systems were recorded throughout the disease evolution. Common presenting manifestations included peripheral thrombosis, neurologic, cardiac, pulmonary, cutaneous and ophthalmic symptoms [7, 8] (Figure 2). Increased incidence of thrombosis, pregnancy loss, valvular heart disease, arthritis, livedo reticularis, renal lesions, cognitive impairment, and cytopenia (thrombocytopenia, leucopenia, hemolytic anemia) was present in patients with SLE-related APS compared to SLE patients without APS $[8,15]$.

Systemic lupus erythematosus patients face an increased risk of developing premature menopause, osteoporotic fractures, decline in cognitive dysfunction, premature atherosclerosis, thrombosis, and cardiovascular events [16]. Yamakami et al. reported diminished ovarian reserve in APS patients [17]. Systemic lupus erythematosus patients frequently present an overlap of menopausal symptoms during disease flares (sleep disturbances, fatigue, difficulty in concentrating, headaches). The effects of menopause in addition to the disease complication lead to a reduced quality of life. Although studies have reported an increased prevalence of premature menopause and menopausal symptoms in women with SLE, no correlation was found between lupus activity and menopausal symptoms or age at menopause onset [6].

The occurrence of thrombosis has been reported in up to $16 \%$ of SLE patients $[18,19]$. Research has shown an even higher frequency among patients with an associated APS [20]. This complication is a primary concern which will affect the disease prognosis and treatment option in patients, especially those in the post-meno- 


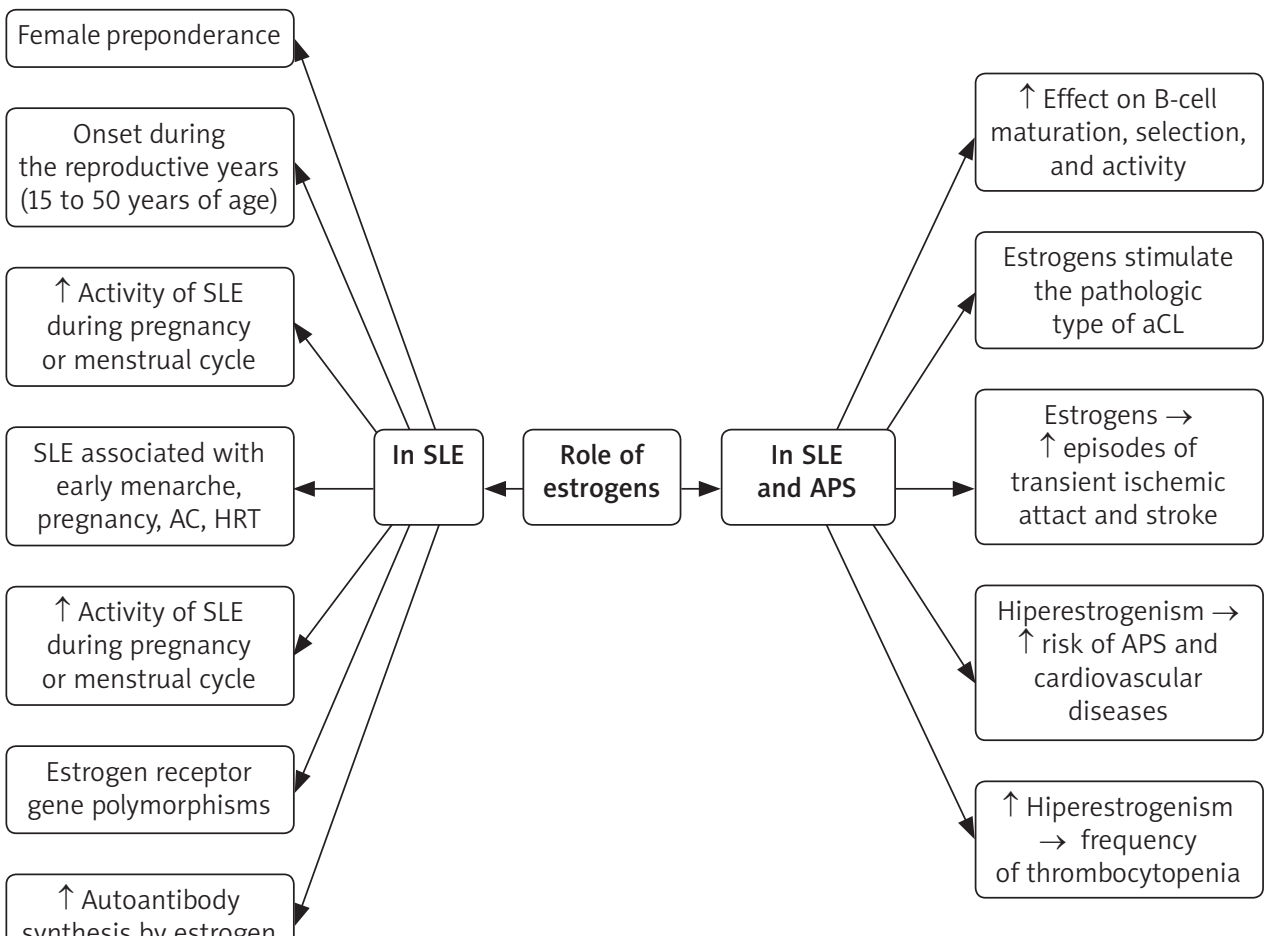

APS - antiphospholipid syndrome, AC - oral contraceptive, HRT - hormone replacement therapy, aCL - anti-cardiolipin antibody

Fig. 1. The role of estrogens in systemic lupus erythematosus (SLE) and SLE-associated antiphospholipid syndrome

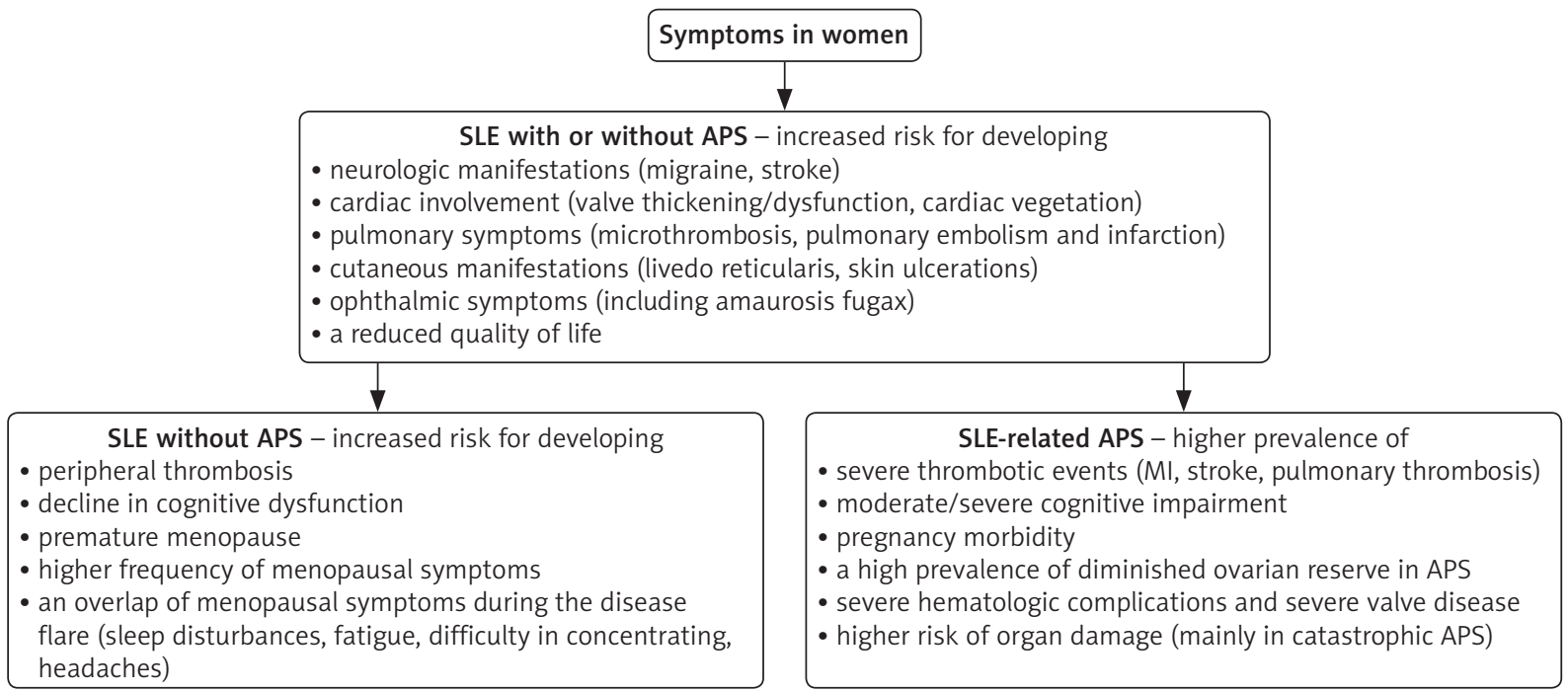

Fig. 2. Signs and symptoms of systemic lupus erythematosus (SLE) and SLE-associated antiphospholipid syndrome (APS) in women

pausal period requiring hormonal replacement therapy, which predisposes them to a higher risk of thrombotic complications [21].

\section{Disease assessment and monitoring}

The complex interplay between SLE and APS reflects the multifaceted management of the combined conditions. The management of APS-associated SLE in peri- and postmenopausal women should begin with a global evaluation and risk stratification of the patient followed by thromboprophylaxis efforts. This involves the correction of modifiable risk factors, lifestyle recommendations, and pharmacological reduction of thrombotic risks [22].

Although the hallmark of pregnancy morbidity is not a primary concern in women in the postmenopausal age group, evaluating the risk of thrombosis and developing strategies to prevent thromboembolic complications are essential. An early evaluation should consider cardiovascular risk factors (hypertension, diabetes mellitus) and factors promoting thrombosis 
(smoking, surgery, immobilization) in addition to the patient's older age [22]. A study by Kaiser et al. of 1930 SLE subjects found that smoking, prolonged disease duration, nephritis, antiphospholipid (aPL) positivity, and immunomodulation medication use were significant risk factors for thrombosis [23].

\section{Lifestyle modifications}

Patients with APS-related SLE can benefit from nonpharmacological lifestyle modifications and diet which may help to decrease their risk of cardiovascular complications [24]. Modifiable risk factors including smoking and a sedentary lifestyle should be identified and health adjustments should be promoted [22]. Comorbid conditions including hypertension, hyperlipidemia, and diabetes mellitus should be strictly monitored and treated [25]. Regular physical activity is beneficial in maintaining the ideal body weight as well in reducing cardiovascular complications [22].

\section{Pharmacological management}

The fundamental principle of APS treatment is thromboprophylaxis using antiplatelet and anticoagulant agents. Patients with venous events should be treated with long-term oral anticoagulation to an internationalized normal ratio (INR) of 2.0-3.0 [26]. In situations of high thrombotic risk, including surgery and prolonged immobilization, low molecular weight heparin (LMWH) should be prescribed [22].

Low dose aspirin (LDASA) and hydroxychloroquine (HCQ) can be used in the reduction of cardiovascular risk and prevention of thrombotic events [22]. Low dose aspirin can be prescribed in individuals with a low-risk aPL profile who develop a stroke. In stroke patients with a higher risk of thrombotic complications, long-term anticoagulation with warfarin should be prescribed [26]. Treatment with hydroxychloroquine was found to be significantly protective against thrombosis through its inhibition of platelet aggregation and aPL production in addition to its cholesterol-lowering mechanisms [23].

\section{Risks and benefits of hormone replacement therapy}

The use of hormone replacement therapy (HRT) in patients with APS-related SLE should be measured against their risk of thrombotic and cardiovascular complications. A prompt initiation should be considered for an increased benefit for bone protection in cases where menopausal symptoms necessitate HRT. The benefit of HRT was observed predominantly in vasomotor symptoms (hot flashes, sweating at night) and not in other menopausal symptoms $[6,27]$. The shortest possible duration of treatment in patients with APS-related SLE should be recommended [28].

Due to research limitations of randomized control trials which included predominantly patients with negative aPL, definite conclusions cannot be made regarding the safety and efficacy of HRT use in APS-related SLE. Among SLE subjects, there have been no reports which demonstrate an increased risk of severe exacerbations within 24 months of HRT, though an increased risk of minor to moderate flares may be observed $[28,29]$.

\section{Conclusions}

APS-related SLE encompasses a heightened risk of thrombotic complications, leading to an increased mortality risk and decreased quality of life in periand postmenopausal patients. The interplay between genetic predisposition, hormonal influence, and environmental factors contributes to the complex clinical image, requiring a multi-step approach. The disease management involves the combination of risk stratification followed by thromboprophylaxis efforts through correction of modifiable risk factors, lifestyle recommendations, and pharmacological treatment.

\section{Disclosure}

The authors report no conflict of interest.

\section{References}

1. Gómez-Puerta JA, Cervera R. Diagnosis and classification of the antiphospholipid syndrome. J Autoimmun 2014; 48-49: 20-25.

2. Singh NK, Agrawal A, Singh MN, et al. Prevalence and pattern of antiphospholipid antibody syndrome in a hospital based longitudinal study of 193 patients of systemic lupus erythematosus. J Assoc Physicians India 2013; 61: 623-626.

3. Franco JS, Molano-González N, Rodríguez-Jiménez M, et al. The coexistence of antiphospholipid syndrome and systemic lupus erythematosus in Colombians. PLoS One 2014; 9: e110242.

4. Mok CC, Chan PT, Ho LY, et al. Prevalence of the antiphospholipid syndrome and its effect on survival in 679 Chinese patients with systemic lupus erythematosus: a cohort study. Medicine (Baltimore) 2013; 92 : 217-222.

5. Urowitz MB, Gladman DD, Tom BD, et al. Changing patterns in mortality and disease outcomes for patients with systemic lupus erythematosus. J Rheumatol 2008; 35: 2152-2158.

6. Cravioto MD, Durand-Carbajal M, Jiménez-Santana L, et al. Efficacy of estrogen plus progestin on menopausal symptoms in women with systemic lupus erythematosus: a randomized, double-blind, controlled trial. Arthritis Care Res (Hoboken) 2011; 63: 1654-1663.

7. García-Carrasco M, Galarza C, Gómez-Ponce M, et al. Antiphospholipid syndrome in Latin American patients: clinical and immunologic characteristics and comparison with European patients. Lupus 2007; 16: 366-373.

8. Cervera R, Boffa MC, Khamashta MA, et al. The Euro-Phospholipid project: epidemiology of the antiphospholipid syndrome in Europe. Lupus 2009; 18: 889-893.

9. Arachchillage DRJ, Laffan M. Pathogenesis and management of antiphospholipid syndrome. Br J Haematol 2017; 178: 181-195.

10. McMurray RW, May W. Sex hormones and systemic lupus erythematosus: review and meta-analysis. Arthritis Rheum 2003; 48: 2100-2110. 
11. Kaliterna DM, Radić M, Ljutić D. Does estrogen stimulate the pathogenic sort of anticardiolipin antibodies? Isr Med Assoc J 2014; 16: 197-198.

12. Costenbader KH, Feskanich D, Stampfer MJ, et al. Reproductive and menopausal factors and risk of systemic lupus erythematosus in women. Arthritis Rheum 2007; 56: 1251-1262.

13. Vinet E, Lee J, Pineau C, et al. Oral contraceptives and risk of systemic lupus erythematosus. Int J Clin Rheumatol 2010; 5: 169-175.

14. Lee YJ, Shin KS, Kang SW, et al. Association of the oestrogen receptor alpha gene polymorphisms with disease onset in systemic lupus erythematosus. Ann Rheum Dis 2004; 63: 1244-1249.

15. Ünlü O, Zuily S, Erkan D. The clinical significance of antiphospholipid antibodies in systemic lupus erythematosus. Eur J Rheumatol 2016; 3: $75-84$.

16. Sánchez-Guerrero J, González-Pérez M, Durand-Carbajal M, et al. Menopause hormonal therapy in women with systemic lupus erythematosus. Arthritis Rheum 2007; 56: 3070-3079.

17. Yamakami LY, Serafini PC, de Araujo DB, et al. Ovarian reserve in women with primary antiphospholipid syndrome. Lupus 2014; 23: 862-867.

18. Calvo-Alén J, Toloza SM, Fernández M, et al. Systemic lupus erythematosus in a multiethnic US cohort (LUMINA). XXV. Smoking, older age, disease activity, lupus anticoagulant, and glucocorticoid dose as risk factors for the occurrence of venous thrombosis in lupus patients. Arthritis Rheum 2005; 52: 2060-2068

19. Sarabi ZS, Chang E, Bobba R, et al. Incidence rates of arterial and venous thrombosis after diagnosis of systemic lupus erythematosus. Arthritis Rheum 2005; 53: 609-612.

20. Farmer-Boatwright MK, Roubey RA. Venous thrombosis in the antiphospholipid syndrome. Arterioscler Thromb Vasc Biol 2009; 29: 321-325.

21. Bergendal A, Kieler H, Sundström A, et al. Risk of venous thromboembolism associated with local and systemic use of hormone therapy in peri- and postmenopausal women and in relation to type and route of administration. Menopause 2016; 23: 593-599.

22. Pons-Estel GJ, Andreoli L, Scanzi F, et al. The antiphospholipid syndrome in patients with systemic lupus erythematosus. J Autoimmun 2017; 76: 10-20.

23. Kaiser R, Cleveland CM, Criswell LA. Risk and protective factors for thrombosis in systemic lupus erythematosus: results from a large, multiethnic cohort. Ann Rheum Dis 2009; 68: 238-241.

24. Soltész P, Szekanecz Z, Kiss E, et al. Cardiac manifestations in antiphospholipid syndrome. Autoimmun Rev 2007; 6: 379-386.

25. Keswani SC, Chauhan N. Antiphospholipid syndrome. J R Soc Med 2002; 95: 336-342.

26. Chighizola CB, Ubiali T, Meroni PL. Treatment of Thrombotic Antiphospholipid Syndrome: The Rationale of Current Management - An Insight into Future Approaches. J Immunol Res 2015; 2015: 951424.

27. Grygiel-Górniak B, Puszczewicz MJ. The influence of endogenous and exogenous sex hormones on systemic lupus erythematosus in pre- and postmenopausal women. Prz Menopauzalny 2014; 13: 262-266.

28. Andreoli L, Bertsias GK, Agmon-Levin N, et al. EULAR recommendations for women's health and the management of family planning, assisted reproduction, pregnancy and menopause in patients with systemic lupus erythematosus and/or antiphospholipid syndrome. Ann Rheum Dis 2017; 76: 476-485.

29. Khafagy AM, Stewart KI, Christianson MS, et al. Effect of menopause hormone therapy on disease progression in systemic lupus erythematosus: a systematic review. Maturitas 2015; 81: 276-281. 